\title{
MIR433 Pre-miRNA
}

National Cancer Institute

\section{Source}

National Cancer Institute. MIR433 Pre-miRNA. NCI Thesaurus. Code C82832.

MIR433 is an oligoribonucleotide that is encoded by the human MIR433 gene and has a role in the regulation of gene expression. 